\title{
Radio-continuum surveys with SKA and LOFAR: a first look at the perspectives for radio mini-halos
}

\author{
M. Gitti ${ }^{1,2}$, G. Brunetti ${ }^{2}$, R. Cassano ${ }^{2}$, and S. Ettori ${ }^{3,4}$ \\ 1 Dipartimento di Fisica e Astronomia (DIFA), Università di Bologna, via Gobetti 93/2, 40129 Bologna, Italy \\ e-mail: myriam.gitti@unibo.it \\ 2 Istituto Nazionale di Astrofisica (INAF) - Istituto di Radioastronomia (IRA), via Gobetti 101, 40129 Bologna, Italy \\ 3 Istituto Nazionale di Astrofisica (INAF) - Osservatorio di Astrofisica e Scienza dello Spazio (OAS), via Gobetti 93/3, 40129 \\ Bologna, Italy \\ ${ }^{4}$ Istituto Nazionale di Fisica Nucleare (INFN) - Sezione di Bologna, viale Berti Pichat 6/2, 40127 Bologna, Italy
}

Received 1 February 2018 / Accepted 14 June 2018

\begin{abstract}
Context. Diffuse synchrotron radio emission has been observed in a number of cool-core clusters on scales comparable to that of the cooling region. These radio sources are called "mini-halos". In order to understand their origin, which is still unclear, joint radio and $\mathrm{X}$-ray statistical studies of large cluster samples are necessary to investigate the radio mini-halo properties and their connection with the cluster thermodynamics.

Aims. We here extend our previous explorative study and investigate the perspectives offered by surveys in the radio continuum with the LOw Frequency ARray (LOFAR) and the Square Kilometre Array (SKA), in particular examining the effect of the intracluster magnetic field in the mini-halo region for the first time.

Methods. By considering the minimum flux detectable in radio surveys and exploiting the $P_{\text {radio }}-L_{\mathrm{X}}$ correlation observed for known mini-halos, we estimate the detection limits achievable by future radio observational follow-up of X-ray cluster samples, such as HIFLUGCS and eROSITA. This allows us to estimate the maximum number of radio mini-halos that can potentially be discovered in future surveys as a function of redshift and magnetic field strength.

Results. Under the optimistic assumption that all cool-core systems host a mini-halo and that the radio versus X-ray scaling relation extends to systems with lower X-ray luminosity, we show that future radio surveys with LOFAR and SKA1 (at $\sim 140 \mathrm{MHz}$ and $\sim 1.4 \mathrm{GHz}$ ) have the potential to discover $\sim 1000-10000$ radio mini-halo candidates up to redshift $z=1$. This shows that these surveys may be able to produce a breakthrough in the study of these sources. We further note that future SKA1 radio surveys at redshift $z>0.6$ will allow us to distinguish between different magnetic fields in the mini-halo region, because higher magnetic fields are expected to produce more powerful mini-halos, thus implying a larger number of mini-halo candidates detected at high redshift. For example, the non-detection with SKA1 of mini-halos at $z>0.6$ will suggest a low magnetic field $(B<$ few $\mu \mathrm{G})$. The synergy of these radio surveys with future $\mathrm{X}$-ray observations and theoretical studies is essential in establishing the radio mini-halo physical nature.
\end{abstract}

Key words. galaxies: clusters: general - radio continuum: galaxies - X-rays: galaxies: clusters

\section{Introduction}

Radio mini-halos are diffuse, faint (at a level of few $\mu \mathrm{Jy} \operatorname{arcsec}^{-2}$ at $1.4 \mathrm{GHz}$ ), amorphous (almost roundish) radio sources with a steep $(\alpha>1$, where the flux density at the frequency $v$ is $S_{v} \propto v^{-\alpha}$ ) radio spectral index (e.g., Feretti et al. 2012, for an observational review). They are extended on scales (total size) $\sim 100-500 \mathrm{kpc}$ at the center of a number of cool-core (CC) clusters, tracing regions where the cooling time of the intracluster medium (ICM) is short. The mini-halo diffuse emission is always observed to surround the intense radio emission of the brightest cluster galaxy (BCG), which often shows nonthermal radio jets and lobes ejected by the central active galactic nucleus (AGN). The lobe radio plasma interacts strongly with the ICM, which is clearly spatially separated from it, by inflating large X-ray cavities and triggering the so-called "radio-mode AGN feedback" (e.g., Gitti et al. 2012, for a review). Although the central radioloud AGN is likely to play a role in the initial injection of the relativistic particles emitting in the mini-halo region, the diffuse radio emission is thought to be not directly connected with the $\mathrm{BCG}$ radio bubbles, but is believed to be truly generated from the
ICM on larger scales, where the thermal and nonthermal components are mixed (e.g., Brunetti \& Jones 2014, for a review).

Similarly to the well-known case of giant halos extended on cluster-scale, the problem of the origin of radio mini-halos stems from the fact that the time necessary for the emitting electrons to diffuse efficiently on the scales covered by the radio emission is longer than their radiative lifetime. To overcome this problem, in situ particle reacceleration by turbulence in the CC region (Gitti et al. 2002, 2004; Mazzotta \& Giacintucci 2008; ZuHone et al. 2013), or alternatively, hadronic models in which secondary electrons are continuously generated by $p-p$ collisions in the cluster volume (Pfrommer \& Enßlin 2004; Jacob \& Pfrommer 2017), have been invoked (Brunetti \& Jones 2014 , for a review).

Nevertheless, the origin of mini-halos is still debated since several fundamental questions remain unresolved. For instance, the possible connection between the origin of mini-halos and the heating of the gas in the central region of $\mathrm{CC}$ clusters is still an open issue. Turbulence in CCs may be generated by several mechanisms, including the interplay between the outflowing relativistic plasma in AGN jets and lobes, and sloshing gas motions 
(Fujita et al. 2004; Heinz et al. 2006; Mazzotta \& Giacintucci 2008; ZuHone et al. 2013; Zhuravleva et al. 2016). Interestingly, turbulence has been measured in the Perseus cluster with properties (strength and scales) that appear compatible with those necessary to explain the origin of the minihalo with reacceleration models (Hitomi Collaboration 2016, 2018). At the same time, turbulent heating has been suggested to play an important role in the thermal balance of galaxy cluster plasmas (Dennis \& Chandran 2005; Gaspari et al. 2012; Zhuravleva et al. 2014). This raises the fascinating possibility of a common origin of radio mini-halos and gas heating in $\mathrm{CC}$, provided that the same turbulence is channelled partly into gas heating and partly into reacceleration of relativistic particles (Bravi et al. 2016). Conversely, the same problem of the origin of mini-halos and the heating of gas in CCs can be addressed in hadronic models through the dissipation of cosmicray induced instabilities into the ICM (Fujita \& Ohira 2012; Jacob \& Pfrommer 2017). In contrast to the case of giant radio halos, the gamma-rays induced by the decay of neutral pions that are generated in hadronic models for mini-halos is still compatible with current gamma-ray limits derived for nearby CCs (e.g., Ahnen et al. 2016; Jacob \& Pfrommer 2017).

Another open problem is generally the connection between mini-halos and gas dynamics of the $\mathrm{CC}$ and of the hosting clusters. Signatures of minor dynamical activity have been detected in a number of clusters hosting mini-halos (Gitti et al. 2007; Cassano et al. 2008), and a morphological connection between mini-halos and cold fronts has been shown in several cases (e.g., Mazzotta \& Giacintucci 2008; Giacintucci et al. 2014a; Gendron-Marsolais et al. 2017). Furthermore, large-scale radio emission in the form of giant radio halos is also detected in a few CC clusters showing minor dynamical activity (e.g., Sommer et al. 2017), suggesting a possible connection or evolution between giant halos and mini-halos (Zandanel et al. 2014; Brunetti \& Jones 2014).

Unfortunately, current observations of mini-halos do not allow us to address these open questions. Obviously, one problem is the limited statistics that prevent us from drawing firm conclusions on the connection between thermal and nonthermal phenomena in CCs. For this reason, Gitti et al. (2015) used scaling relations between the properties of mini-halos and of the hosting CC clusters to start investigating the capabilities of the Square Kilometre Array (SKA, Braun et al. 2015) in the detection of mini-halos at mid frequencies $(\sim 1 \mathrm{GHz})$.

In this paper, we extend the previous work to low radio frequencies and derive expectations assuming different magnetic fields in the mini-halo region. We focus in particular on the case of the LOw Frequency ARray (LOFAR, van Haarlem et al. 2013) and SKA-LOW at $\sim 140 \mathrm{MHz}$, and also discuss the synergy with current and future X-ray missions such as eROSITA (Pillepich et al. 2012). The questions we would like to address are essentially whether these next-generation radio telescopes may be expected to significantly increase the statistics of these radio sources, detecting mini-halos in less X-ray luminous systems and at higher redshift, and to constrain the intracluster magnetic field in the mini-halo region.

We adopt a $\Lambda$ cold dark matter cosmology with $H_{0}=70 \mathrm{~km} \mathrm{~s}^{-1} \mathrm{Mpc}^{-1}, \Omega_{\mathrm{M}}=1-\Omega_{\Lambda}=0.3$.

\section{Mini-halo sample: current statistics}

The detection of radio mini-halos is complicated by the need to separate their faint, low surface brightness emission from the bright emission of the radio-loud BCG embedded in it. This

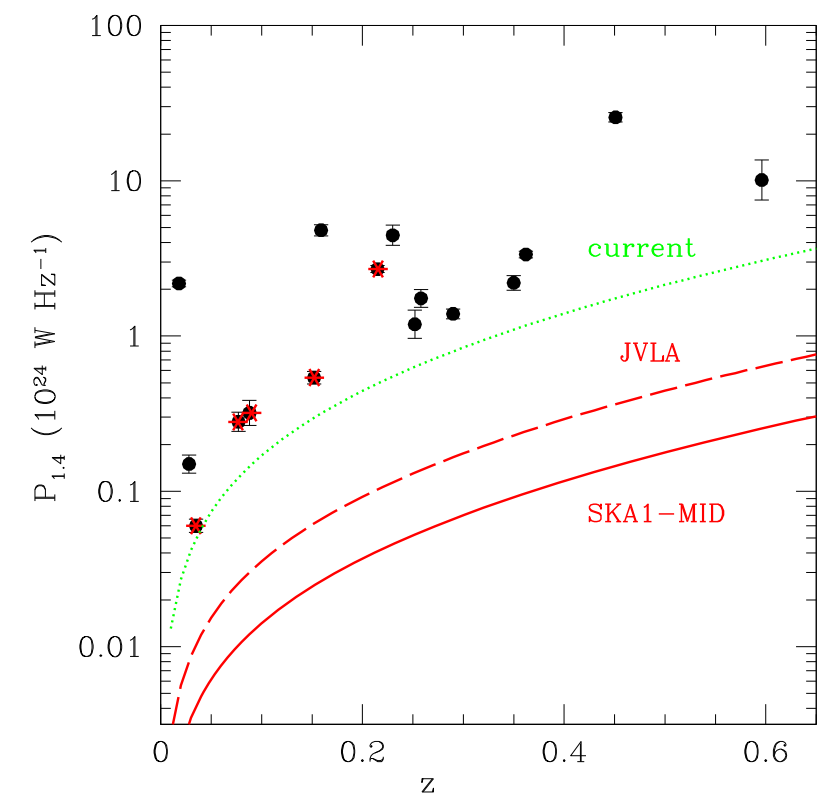

Fig. 1. Radio power at $1.4 \mathrm{GHz}$ (from Giacintucci et al. 2014b; van Weeren et al. 2014) versus redshift of the sample of 16 confirmed mini-halos known up to 2016 (sorted by decreasing radio power at $1.4 \mathrm{GHz})$ : RXJ1347.5-1145 $(z=0.451)$, Phoenix $(z=0.596)$, RXJ1720.1+2638 $(z=0.159)$, A $2390(z=0.23)$, RXJ1532.9+3021 $(z=0.362), \quad$ RXC J1504.1-0248 $(z=0.215), \quad$ RBS $797 \quad(z=0.35)$, Perseus $\quad(z=0.018), \quad$ MS 1455.0+2232 $\quad(z=0.258), \quad \mathrm{ZwCl} 3146$ $(z=0.290)$, A $1835(z=0.252)$, A $2204(z=0.152)$, A $478(z=0.088)$, A $2029(z=0.077)$, Ophiuchus $(z=0.028)$, and 2A 0335+096 $(z=0.035)$. The clusters belonging to the HIFLUGCS sample are highlighted with red stars. The green dotted line is indicative of the existing detection limit reached in the literature with pointed observations. The red dashed and solid lines are the detection limits achievable with JVLA and SKA1-MID, respectively, with the performances adopted in Table 1 (see Sect. 3.1).

requires observations performed with a technical setup ensuring high sensitivity to diffuse emission, provided by short baselines, but at the same time allowing an accurate subtraction of discrete sources, which are best identified by long baselines. Therefore, good spatial dynamic ranges, along with dynamic ranges much higher $(\gg 1000)$ than those in available surveys, are typically required to properly image the radio mini-halo emission. For this reason, the mini-halo detection has indeed proven to be very challenging with the current facilities, even with pointed observations (e.g., Govoni et al. 2009; Giacintucci et al. 2014b). A first statistical assessment of the occurrence of mini-halos in a mass-selected $\left(M_{500}>6 \times 10^{14} M_{\odot}\right)$ sample of galaxy clusters found that all mini-halos are hosted by CC clusters, defined as clusters with a value of the central entropy $K_{0}<30 \mathrm{keV} \mathrm{cm}^{2}$ (where $K_{0}=k T_{0} n_{0}^{-2 / 3}, n_{0}$ and $T_{0}$ being the central values of the ICM electron density and temperature, respectively), and that almost all CC clusters of the sample, namely $\sim 80 \%$, host a minihalo (Giacintucci et al. 2017). This fraction is similar to the fraction of CC clusters whose BCG hosts a radio AGN (70\%, Burns 1990; Dunn \& Fabian 2006; Best et al. 2007; Mittal et al. 2009), in line with the observed evidence that AGN are ubiquitous in mini-halos (e.g., Giacintucci et al. 2014b).

In Fig. 1 we show the $K$-corrected radio power at $1.4 \mathrm{GHz}$ versus redshift distribution of the sample of confirmed minihalos known up to 2016, which comprises 16 objects selected from the mini-halo collection reported in Giacintucci et al. (2014b, and references therein) by excluding the objects that 
they classify as "candidate" or "uncertain" and including the new detection in the Phoenix cluster (van Weeren et al. 2014). We quantitatively checked that the 16 mini-halos in Fig. 1 are hosted in CC clusters; by taking values from the literature (Cavagnolo et al. 2011; Hudson et al. 2010; McDonald et al. 2012), we find that all mini-halo clusters have central entropy $K_{0} \lesssim 25 \mathrm{keV} \mathrm{cm}^{2}$. According to Hudson et al. (2010), this definition applies to strong cool-core (SCC) clusters. The green dotted line in Fig. 1 is indicative of the current mini-halo detection limit obtained by assuming angular resolution $\theta_{\mathrm{b}}=5 \operatorname{arcsec}$ and sensitivity $=30 \mu \mathrm{Jy} \mathrm{beam}^{-1}$, typical of pointed observations with the VLA in B-array (see Sect. 3.1 for details on the criterion adopted to derive the minimum detectable flux). It is evident that our current ability of detecting mini-halos is limited and that we may be missing many faint mini-halos.

One possibility for estimating the perspective offered by the SKA and its pathfinders in the ability of detecting radio mini-halos is to first link the nonthermal properties of these sources to the thermal properties of their host galaxy clusters. For the above sample of 16 confirmed mini-halos, we considered the X-ray luminosity taken from the MCXC catalog of (Piffaretti et al. 2011; for Phoenix, which is the only cluster not included in the MCXC, the luminosity was taken from McDonald et al. 2012). This catalog is based on publicly available ROSAT All Sky Survey data, which have then been systematically homogenized to an overdensity of 500 . We converted the tabulated X-ray luminosity from $0.1-2.4 \mathrm{keV}$ into $0.5-2 \mathrm{keV}$ band assuming an Xspec apec plasma model at the observed cluster temperature, redshift, and metallicity (taken from Cavagnolo et al. 2009; Hudson et al. 2010). In these energy bands, the signal is almost independent of any temperature and is only proportional to the square of the gas density. Therefore, the conversion itself introduces a negligible systematic error in the total error budget of about $10 \%$ that we propagate through our statistical analysis. The resulting average conversion factor for our sample is $L_{0.5-2.0} \sim 0.6 L_{0.1-2.4}$. The choice of this particular energy band was made for consistency with the method used in Sect. 3.3 to estimate the radio luminosity function of minihalos by linking it to the X-ray luminosity function of galaxy clusters, which is measured in the $0.5-2.0 \mathrm{keV}$ energy band (Mullis et al. 2004).

We found a correlation between the $1.4 \mathrm{GHz}$ radio power of the mini-halos, $P_{1.4}$ (in units of $10^{24} \mathrm{~W} \mathrm{~Hz}^{-1}$ ), and the global $\mathrm{X}$-ray luminosity of the host clusters in the $0.5-2.0 \mathrm{keV}$ band, $L_{\mathrm{X}}$ (in units of $10^{44} \mathrm{erg} \mathrm{s}^{-1}$ ), in the form

$\log P_{1.4}=a \log L_{\mathrm{X}}+b$,

where $a=2.03 \pm 0.20$ and $b=-1.65 \pm 0.21$ are the best-fit values derived by using the bivariate correlated error and intrinsic scatter (BCES) algorithm (Akritas \& Bershady 1996) to perform regression fits (bisector method). The best-fit correlation is shown in Fig. 2, along with the lines enclosing the area that has a $1 \sigma$ chance of containing the true regression line (estimated from Eq. 7 in Cassano et al. 2013).

To ensure that the trend observed in the radio luminosity - X-ray luminosity plane is not biased by any effect due to the underlying dependence of these two properties on redshift, we checked that a correlation is present in the radio flux X-ray flux plane as well. We found a Spearman rank correlation coefficient of $r_{s} \sim 0.6$ in both planes, with a probability of no correlation of probs $\sim 1 \%$, thus indicating that the relation between mini-halo radio power and X-ray luminosity may be intrinsic.

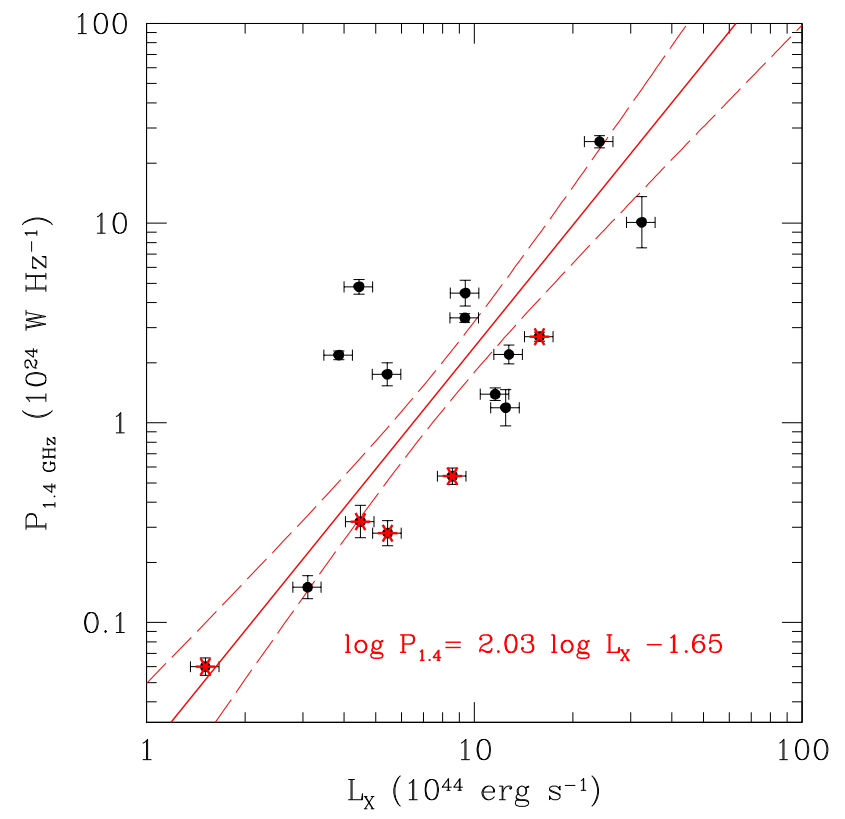

Fig. 2. Radio power at $1.4 \mathrm{GHz}$ versus the X-ray luminosity inside $R_{500}$ (taken from Piffaretti et al. 2011, and converted into the $0.5-2.0 \mathrm{keV}$ band) for the mini-halo sample. The red solid line is the best-fit relation (see Eq. (1)), enclosed by the $1 \sigma$ confidence lines (red dashed). The clusters belonging to the HIFLUGCS sample are highlighted with red stars.

\section{Role of future radio surveys}

\subsection{Detecting radio mini-halos in galaxy clusters}

In order to evaluate the role of future radio surveys in the ability to discover new radio mini-halos, we need to estimate the minimun flux of diffuse radio emission that can be detected with given observational performances. To do so, we assumed an exponential distribution of the diffuse radio brightness (Murgia et al. 2009) and determined the region that contains a significant fraction of the integrated flux density. In particular, assuming a radial profile in the form $I(r)=I_{0} e^{-r / r_{e}}$, where $I_{0}$ is the central radio surface brightness and $r_{e}$ is the $e$-folding radius (i.e., the radius at which the brightness drops to $I_{0} / e$ ), radio mini-halos emit about half of their total radio flux within a radius $r_{\text {half }} \sim 1.68 r_{e}$, which is called the half-radius. In order to estimate the minimum flux that can be detected with observations performed at given resolution and sensitivity levels, we adapted to mini-halos the criterion of Cassano et al. (2012) based on a threshold in flux for giant halos, and assumed that mini-halos can be detected when the integrated flux within their half-radius gives a signal-to-noise ratio $\xi_{2}$. In particular, the minimum detectable flux as a function of redshift, $f_{\min }(z)$, is

$f_{\text {min }}(z) \simeq 2.86 \times 10^{-3} \xi_{2}\left(\frac{F_{\text {rms }}}{10 \mu \mathrm{Jy}}\right)\left(\frac{10^{\prime \prime}}{\theta_{\mathrm{b}}}\right)\left(\frac{\theta_{\text {half }}(z)}{1^{\prime \prime}}\right) \quad[\mathrm{mJy}]$,

where $F_{\text {rms }}$ and $\theta_{\mathrm{b}}$ are the rms noise per beam and the beam angular size (in arcsec) of the observations, respectively, and $\theta_{\text {half }}(z)$ is the angular size (in arcsec) of the mini-halo half radius at a given redshift.

We aim at evaluating the possible detections of mini-halos with a typical size of $\sim 200 \mathrm{kpc}$ (e.g., Giacintucci et al. 2014b), therefore we assume a representative half-radius of $\sim 100 \mathrm{kpc}$. This is in line with the lack of a clear correlation between the mini-halo size and other cluster properties, such as the X-ray 


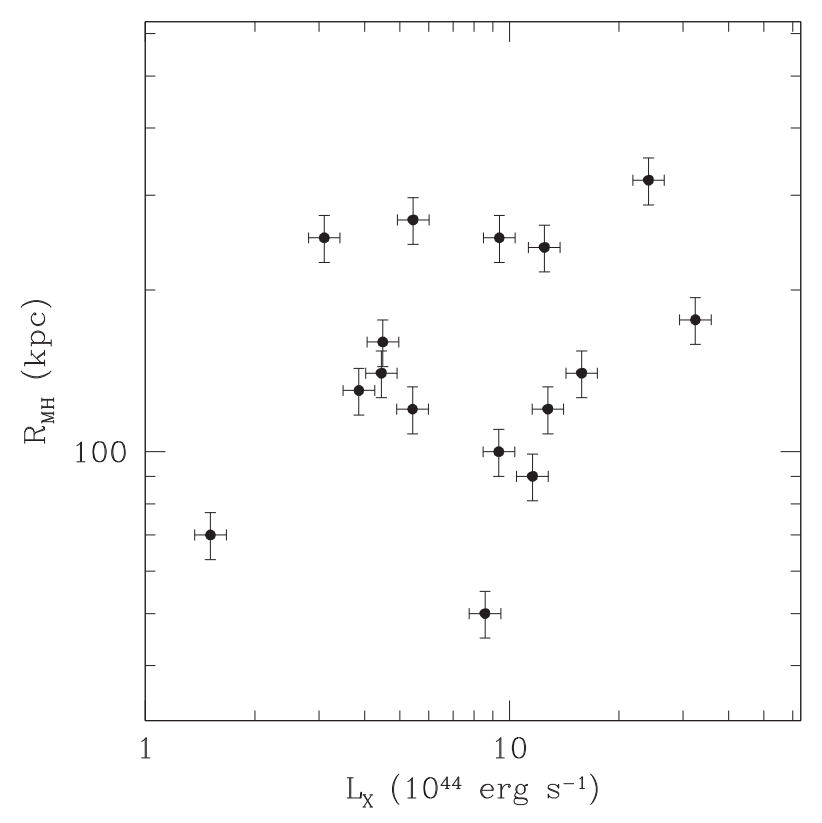

Fig. 3. Average radius of the mini-halo (from Giacintucci et al. 2014b; Bravi et al. 2016) versus the X-ray luminosity inside $R_{500}$ (taken from Piffaretti et al. 2011, and converted into the $0.5-2.0 \mathrm{keV}$ band). No obvious correlation is visible (we found $r_{s} \sim 0.2$, with a probability of no correlation of probs $\sim 40 \%$ ).

luminosity, as shown in Fig. 3. We did not find any obvious correlation between the mini-halo radius and the $1.4 \mathrm{GHz}$ radio power either $\left(r_{s} \sim 0.2\right.$, with a probability of no correlation of probs $\sim 40 \%$ ), which is in agreement with the observed $P_{1.4}-L_{X}$ correlation. Therefore, $\theta_{\text {half }}$ is a function of redshift alone. As an example, at redshift $z=0.6$, the angular half-radius of a typical radio mini-halo is $\theta_{\text {half }} \sim 15$ arcsec. Following Cassano et al. (2015), we further adopt $\xi_{2}=7$ as a reference value.

We here consider the radio observational performances reported in Table 1: at mid frequency, the SKA1-MID all sky survey at $\sim 1.4 \mathrm{GHz}$ (Prandoni \& Seymour 2015), along with JVLA pointed observations in $L$ band (1-2 GHz), and at low frequency, the LOFAR Two-metre Sky Survey (LoTSS, Shimwell et al. 2017) and SKA1-LOW all-sky surveys at $\sim 140 \mathrm{MHz}$ (Prandoni \& Seymour 2015). In particular, we estimated the minimum flux as described above by assuming values of SKA1 surveys at confusion limit (see Cassano et al. 2015, for a more detailed discussion). We did not consider the upcoming VLA Sky Survey (VLASS) ${ }^{1}$ at 2.5 -arcsec resolution because owing to the relatively high frequency ( $S$-band: $2-4 \mathrm{GHz})$ and low sensitivity ( 120 or $70 \mu \mathrm{Jy}_{\text {beam }}{ }^{-1}$ achievable with 3 passes or 1 pass, respectively), it is not expected to be competitive in detecting mini-halos.

The value of $\theta_{\mathrm{b}}$ in Table 1 can be obtained after tapering from higher-resolution images. For example, the angular resolution of a few arcseconds that can be achieved at medium frequency can be used to produce BCG-subtracted images, which can be then tapered up to a lower resolution to increase the sensitivity to the extended emission.

From the minimum detectable flux (Eq. (2)), we estimated the minimum detectable radio power at each redshift calculated as $P_{v}(z)=4 \pi D_{\mathrm{L}}^{2} f_{\min , v}(z)(1+z)^{(\alpha-1)}$, where $D_{\mathrm{L}}$ is the luminosity distance, and $(1+z)^{(\alpha-1)}$ is the $\mathrm{K}$-correction term, thus obtaining

\footnotetext{
1 https://science.nrao.edu/enews/8.11/index.shtml\# vlass
}

Table 1. Observational performances adopted in this work.

\begin{tabular}{lcccc}
\hline \hline Instrument & Mode & $\begin{array}{c}v \\
(\mathrm{MHz})\end{array}$ & $\begin{array}{c}F_{\mathrm{rms}} \\
\left(\mu \mathrm{Jy} \mathrm{beam}^{-1}\right)\end{array}$ & $\begin{array}{c}\theta_{\mathrm{b}} \\
\left({ }^{\prime \prime}\right)\end{array}$ \\
\hline JVLA & Pointed & 1400 & 10 & 8 \\
SKA1-MID & Survey $(3 \pi)$ & 1400 & 4 & 8 \\
LOFAR & Survey $(3 \pi)$ & 140 & 200 & 8 \\
SKA1-LOW & Survey $(3 \pi)$ & 140 & 20 & 8 \\
\hline
\end{tabular}

Notes. $F_{\text {rms }}$ is the sensitivity, $\theta_{\mathrm{b}}$ is the tapered resolution. At $v \sim 1.4 \mathrm{GHz}$, the survey speed of SKA1-MID is more than two orders of magnitude faster than JVLA, whereas at $v \sim 140 \mathrm{MHz}$ the survey speed of SKA1LOW is more than one order of magnitude faster than LOFAR ${ }^{2}$.

a survey sensitivity in terms of radio power. The results on the $1.4 \mathrm{GHz}$ detection limit are shown in Fig. 1, where it is evident that the JVLA represents already a great improvement with respect to existing observations. We note, however, that such performances require pointed observations. According to the JVLA exposure calculator, confirmed by our direct experience on real observations (e.g., Ignesti et al. 2017), the values adopted in Table 1 are achievable with approximately twohour pointings. On the other hand, SKA1-MID surveys will be able to detect all mini-halos with $P_{1.4} \gtrsim 2 \times 10^{23} \mathrm{~W} \mathrm{~Hz}^{-1}$ up to redshift 0.6 .

\subsection{Radio follow-up of $X$-ray cluster samples}

We further aim at investigating the radio follow-up of X-ray cluster samples by adopting the basic assumption that mini-halos follow the observed $P_{1.4}-L_{X}$ correlation (Eq. 1). This correlation indicates a connection between the energy reservoir in $\mathrm{CC}$ clusters and that associated with the nonthermal components powering radio mini-halos (see also Bravi et al. 2016). The nonthermal radiation, $P_{\mathrm{NT}}$, that can be maintained in the region of radio mini-halos for a timescale that is longer than the radiative life-time of the relativistic electrons is

$P_{\mathrm{NT}}=P_{\text {radio }}+P_{\mathrm{IC}}=P_{\text {radio }}\left[1+\left(\frac{B_{\mathrm{CMB}}}{B}\right)^{2}\right]$,

where $P_{\text {radio }}$ is the synchrotron radiation, $P_{\mathrm{IC}}$ is the inverse Compton radiation, $B_{\mathrm{CMB}}=3.25(1+z)^{2} \mu \mathrm{G}$ is the magnetic field equivalent to the inverse Compton losses with $\mathrm{CMB}$ photons, and $B$ is the magnetic field intensity in the mini-halo region.

We can thus express the observed synchrotron radiation as a function of the intracluster magnetic field and redshift: $P_{\text {radio }}=P_{\text {norm }}\left[B^{2} /\left(B^{2}+B_{\mathrm{CMB}}^{2}\right)\right]$, where the normalization $P_{\text {norm }}$ is a function of $B$ and is obtained by imposing that at the mean redshift of our sample $(\langle z\rangle=0.22)$, the synchrotron radiation observed at $1.4 \mathrm{GHz}$ follows Eq. (1).

By matching the observed normalization of the correlation, we obtained the general correlation (where we make the dependencies explicit)

$P_{1.4}(B, z)=10^{b}\left(\frac{B^{2}+23.4}{B^{2}+10.6(1+z)^{4}}\right) L_{\mathrm{X}}^{a}$,

where $P_{1.4}$ is in units of $10^{24} \mathrm{~W} \mathrm{~Hz} \mathrm{~Hz}^{-1}, L_{\mathrm{X}}$ is in units of $10^{44} \mathrm{erg} \mathrm{s}^{-1}, B$ is in $\mu \mathrm{G}$, and $a$ and $b$ are the parameters in Eq. (1).

By combining this correlation with the minimum detectable radio power, estimated in Sect.3.1 with the performances in

2 https://astronomers.skatelescope.org/documents 

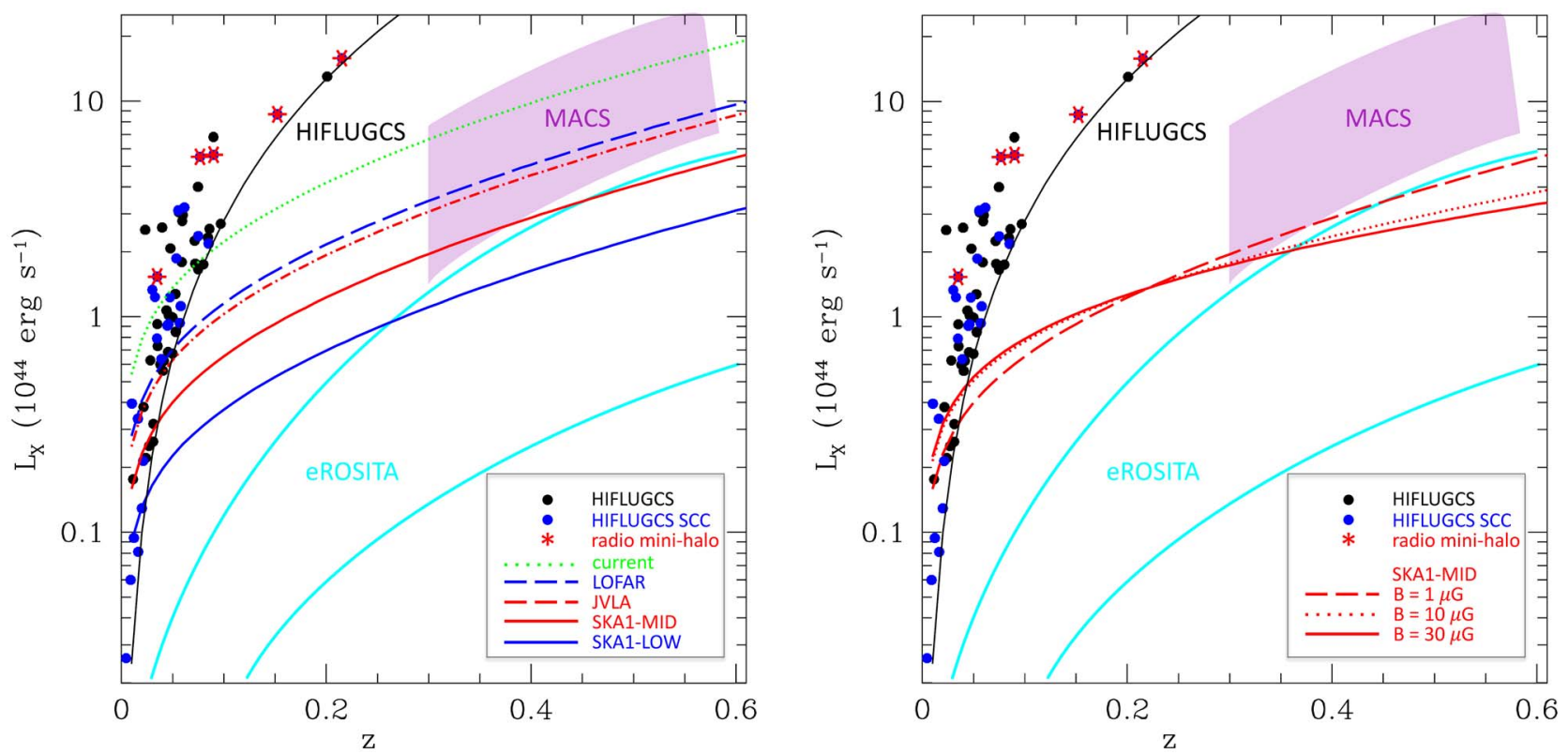

Fig. 4. Left panel: X-ray luminosity in the $0.5-2.0 \mathrm{keV}$ band versus redshift for the clusters in the HIFLUGCS sample (Reiprich \& Böhringer 2002). The flux limit is shown as a black solid line. The blue filled circles represent the clusters classified as SCC (Hudson et al. 2010), and the clusters known to host radio mini-halos are highlighted with red stars. For comparison, we also draw the $L_{\mathrm{X}}-z$ distribution of clusters from other X-ray selected samples: the shaded magenta region indicates the position of MACS clusters (Ebeling et al. 2010), and the cyan solid lines show the eROSITA threshold luminosity predicted with 500 (upper line) and 50 (lower line) photon counts (Pillepich et al. 2012). Our results on the detection limits reachable by radio follow-up are estimated with the performances adopted in Table 1 (see Sect. 3.1) and a typical magnetic field of $B=1 \mu \mathrm{G}$ : from existing observations (green dotted line), from 1.4 GHz JVLA pointed observations and SKA1-MID surveys (red dashed and solid lines, respectively), and from $140 \mathrm{MHz}$ LOFAR and SKA1-LOW surveys (blue dashed and solid lines, respectively). Right panel: same as left panel, but showing only the detection limits reachable by radio follow-up with SKA1-MID assuming three different magnetic fields: $B=1 \mu \mathrm{G}$ (dashed line), $B=10 \mu \mathrm{G}$ (dotted line), and $B=30 \mu \mathrm{G}$ (solid line).

Table 1, we finally obtained the X-ray luminosity thresholds that allow us to investigate the possible radio follow-up coverage of (existing and future) X-ray cluster samples.

In particular, we consider the X-ray HIFLUGCS sample (HIghest X-ray FLUx Galaxy Cluster Sample, Reiprich \& Böhringer 2002), which is a flux-limited $\left(f_{X} \geq 2 \times\right.$ $10^{-11} \mathrm{erg} \mathrm{s}^{-1} \mathrm{~cm}^{-2}$ in the $0.1-2.4 \mathrm{keV}$ ROSAT band) statistically complete sample of 64 galaxy clusters with mean redshift $\langle z\rangle=0.05$, with the largest coverage $(100 \%)$ of high-quality $\mathrm{X}$-ray data from Chandra. We plot in Fig. 4 the distribution of the $\mathrm{X}$-ray luminosity in the $0.5-2.0 \mathrm{keV}$ energy band versus redshift and highlight with red stars the five HIFLUGCS clusters that are known to host a radio mini-halo; we note that all lie on top of the distribution. For comparison, we also show other X-ray selected samples that cover different $L_{X}-z$ distributions: the observed Massive Cluster Survey (MACS, Ebeling et al. 2010), and the predicted eROSITA thresholds (Pillepich et al. 2012).

To derive the minimum X-ray luminosity of clusters with detectable radio mini-halos in low-frequency surveys, we assume that mini-halos have a typical spectral index $\alpha=1.2$ (e.g., Giacintucci et al. 2014b) and that consequently their $140 \mathrm{MHz}$ radio power has the same scaling with the X-ray luminosity as that at $1.4 \mathrm{GHz}$ (Eq. 1). The results for the different survey performances adopted in Table 1 are shown in Fig. 4 (left panel) assuming a typical magnetic field of $\mathrm{B}=1 \mu \mathrm{G}$, and the effect of varying the magnetic field between $\mathrm{B}=1 \mu \mathrm{G}$ and $30 \mu \mathrm{G}$ are shown in Fig. 4 (right panel) for the SKA1-MID survey alone. Our main notes are listed below.

- The radio selection limits have different shapes than the X-ray flux-limited selections. This is important because future radio surveys will allow us to reach much higher redshifts than those of X-ray cluster selections with similar sensitivities in the local universe.

- A consistent fraction of the clusters selected and characterized by eROSITA will be observable by SKA and LOFAR in various redshift ranges. This is important because it will allow us to build large cluster samples that can be exploited to study not only the occurrence of radio mini-halos, but also the radio and X-ray properties with an accuracy sufficient to study the interplay between nonthermal and thermal cluster components.

- Radio surveys can be used to identify the first SCC in the universe, that is, the highest redshift mini-halos with associated X-ray emission (particularly with SKA1-LOW at $z>0.6$, see Sect. 3.3). This may place constraints on the formation of SCC and related radio emission.

\subsection{How many radio mini-halos await discovery?}

We estimated the maximum number of radio mini-halo candidates that might be discovered in future surveys as a function of redshift $z$ by integrating the radio luminosity function (RLF) of mini-halos $\mathrm{d} N_{\mathrm{MH}} /(\mathrm{d} P \mathrm{~d} V)$ over radio luminosity and $z$,

$N_{\mathrm{MH}}^{\Delta z}=\int_{z_{1}}^{z_{2}} \mathrm{~d} z^{\prime}\left(\frac{\mathrm{d} V}{\mathrm{~d} z^{\prime}}\right) \int_{P_{\min }\left(z^{\prime}\right)} \mathrm{d} P \frac{\mathrm{d} N_{\mathrm{MH}}}{\mathrm{d} P \mathrm{~d} V}$,

where the minimum radio luminosity detectable at a given $z$, $P_{\text {min }}$, can be estimated from Eq. (2).

Our basic assumptions to calculate the RLF in Eq. (5) are that (1) every SCC cluster (as defined by Hudson et al. 2010) hosts a radio mini-halo, and this mini-halo follows the observed $P_{\text {radio }}-L_{\mathrm{X}}$ correlation (Eq. (1)), and

(2) the fraction of SCC clusters does not evolve strongly with redshift. 
These are two strong assumptions. The first assumption is supported by Giacintucci et al. (2017) at least for massive systems. These authors indeed found that about $80 \%$ of massive $\mathrm{CC}$ clusters host a mini-halo. On the other hand, the occurrence of mini-halos in less massive CC clusters is currently unconstrained. The second assumption, still debated in the literature (e.g., Santos et al. 2010; Samuele et al. 2011), is motivated for CC systems with a luminosity above a few $\times 10^{44} \mathrm{erg} \mathrm{s}^{-1}$, but current data do not allow us to observe this correlation at lower $\mathrm{X}$-ray luminosities, that is, in clusters that contribute to the number counting of mini-halos from Eq. (5) (see Fig. 4).

Under these assumptions, the RLF of mini-halos per sky area surveyed in steradians is (see Eq. 3.2 of Gitti et al. 2015)

$\frac{\mathrm{d} N_{\mathrm{MH}}}{\mathrm{d} P \mathrm{~d} V}=\mathrm{f}_{\mathrm{SCC}} \frac{\mathrm{d} N_{\mathrm{cl}}}{\mathrm{d} L_{\mathrm{X}} \mathrm{d} V} \frac{\mathrm{d} L_{\mathrm{X}}}{\mathrm{d} P_{\text {radio }}}$,

where $\mathrm{d} N_{\mathrm{cl}} /\left(\mathrm{d} L_{\mathrm{X}} \mathrm{d} V\right)=\phi\left(L_{\mathrm{X}}, z\right)$ is the X-ray luminosity function (XLF) of galaxy clusters, $\mathrm{f}_{\mathrm{SCC}}$ is the fraction of clusters with SCC (we adopted $\sim 0.4$, estimated for the HIFLUGCS sample by Hudson et al. 2010) and $\mathrm{d} L_{\mathrm{X}} / \mathrm{d} P_{\text {radio }}$ is obtained from Eq. (4).

We considered the evolving XLF derived from the highredshift X-ray-selected 160 Square Degree ROSAT Cluster Survey (160SD) by Mullis et al. (2004) in a $\Lambda$-dominated universe. We assumed the local XLF determined from the REFLEX survey, which is the only local sample for which the XLF in a $\Lambda$-cosmology has been explicitly measured (Böhringer et al. 2002), and the evolution estimated through the maximum likelihood analysis using the 66 clusters at $z>0.3$ in the 160SD sample, adopted by Mullis et al. (2004) itself as the less biased results (for more details on the derivation of the analytic XLF, we refer to Mullis et al. 2004).

In particular, we adopted (see Eq. (5) of Mullis et al. 2004, with $\alpha=1.69$ taken from their Table 1)

$$
\phi\left(L_{\mathrm{X}}, z\right)=\phi^{*}(z)\left(\frac{L_{\mathrm{X}}}{L_{\mathrm{X}}^{*}(z)}\right)^{-1.69} \exp \left(-\frac{L_{\mathrm{X}}}{L_{\mathrm{X}}^{*}(z)}\right) \frac{1}{L_{\mathrm{X}}^{*}(z)},
$$

where $\phi^{*}(z)=2.9 \times 10^{-7}\left((1+z) /\left(1+z_{0}\right)\right)^{0.6} \mathrm{Mpc}^{-3}$, and $L_{\mathrm{X}}^{*}(z)=2.6 \times 10^{44}\left((1+z) /\left(1+z_{0}\right)\right)^{-2.1} \mathrm{erg} \mathrm{s}^{-1}, z_{0}$ being the median redshift sampled by the local XLF $\left(z_{0}=0.08\right.$ for the REFLEX survey).

Operatively, for each redshift interval $\left(z_{1}, z_{2}\right)$, we numerically solved the integral in the form

$N_{\mathrm{MH}}^{\Delta z}=\int_{z_{1}}^{z_{2}} \mathrm{~d} z^{\prime}\left(\frac{\mathrm{d} V}{\mathrm{~d} z^{\prime}}\right) \int_{P_{\min }\left(z^{\prime}\right)} \mathrm{d} P_{\text {radio }} f_{\mathrm{SCC}} \phi\left(L_{\mathrm{X}}, z^{\prime}\right) \frac{1}{a} \frac{L_{\mathrm{X}}}{P_{\text {radio }}}$,

where the ratio $L_{\mathrm{X}} / P_{\text {radio }}$ is calculated from Eq. (4), and $a$ is the best-fit parameter of the correlation in Eq. (1).

The uncertainty on $N_{\mathrm{MH}}^{\Delta z}$ is mainly driven by the $P_{\text {radio }}-L_{\mathrm{X}}$ correlation. The internal accuracy of the XLF measurements is approximately $\pm 10 \%-20 \%$ (estimated from the $\pm 1 \sigma$ excursion of the error envelopes in Fig. 4 of Mullis et al. 2004), and the systematics are also small. For example, the results of the BCS survey (Ebeling et al. 1997) vary by a maximum of about $\pm 25 \%$ relative to the Schechter fit of the REFLEX assumed in this work (Mullis et al. 2004). Therefore, to estimate the uncertainty on $N_{\mathrm{MH}}^{\Delta z}$ we fixed the cluster XLF and performed Monte Carlo simulations by considering 2000 random choices of the parameters $a$ and $b$, and report the $1 \sigma$ error.

We calculated the integral number of expected radio minihalo candidates as a function of redshift by assuming $B$ between 1 and $30 \mu \mathrm{G}$. Our predictions at $1.4 \mathrm{GHz}$ (with SKA1-MID) and $140 \mathrm{MHz}$ (with LOFAR and SKA1-LOW) are shown in Fig. 5 (top left panel). In particular, we estimated that all-sky surveys with SKA1 may be able to detect up to $\sim 4,000$ and $\sim 10^{4}$ new mini-halo candidates out to redshift $z \sim 1$ at -MID and -LOW frequency, respectively. While we wait for SKA, its pathfinder LOFAR may already have the capabilities to discover up to $\sim 1,400$ new mini-halo candidates, which represents an enormous improvement with respect to the current statistics.

These numbers refer to the assumption that all $\mathrm{CC}$ clusters host a mini-halo, regardless of the mass and dynamical status of these clusters. In the context of hadronic models, this is probably reasonable at some level. On the other hand, in the case of reacceleration or leptonic models, the possibility of maintaining radio-emitting electrons in the $\mathrm{CC}$ region depends on the level of turbulence and on the fraction of the turbulent energy flux that is damped into particle reacceleration. If this turbulence is generated by perturbations in the core that are induced by dynamics on larger scales (e.g., ZuHone et al. 2013), we may expect that the turbulent level declines in less massive and less dynamically disturbed systems. This will induce a declining fraction of minihalos in CC, and consequently, the number density of mini-halos would be significantly smaller than that expected from our simple calculations. Although this adds substantial uncertainties in the model expectations, it is also true that a comparison between our simple expectations and future surveys is a unique way to readily pinpoint the role played by turbulence and reacceleration mechanisms in mini-halos, and raises the possibility of distinguishing between different origins of these radio sources.

In Fig. 5 (top right, bottom left, and bottom right panels) we report the differential number of mini-halos expected to be observable in different redshift bins at $1.4 \mathrm{GHz}$ (by SKA1MID), and at $140 \mathrm{MHz}$ (by LOFAR and SKA1-LOW), respectively, estimated by assuming three reference values of $B=1 \mu \mathrm{G}$, $B=10 \mu \mathrm{G}$, and $B=30 \mu \mathrm{G}$.

Model expectations significantly differ at redshift higher than 0.6. The reason is that in the case of weak magnetic fields an increasing fraction of the nonthermal luminosity is channelled into inverse Compton emission at higher redshifts, making minihalos progressively less luminous and thus more difficult to observe. Based on our calculations, the future SKA1 radio surveys will allow us to distinguish among different assumptions on the magnetic field intensities in the mini-halo region. For example, an SKA1-MID detection of more than 10 mini-halos at $z>0.6$ will suggest a stronger magnetic field $(B \geq 10-30 \mu \mathrm{G})$, but an SKA1-LOW non-detection of mini-halos above the same redshift will indicate a lower value of $B<1 \mu \mathrm{G}$. On the other hand, LOFAR observations are already able to provide some constraints. For example, a LOFAR detection of more than 20 mini-halos in the redshift bin $z=0.4-0.6$ will suggest $B \geq 30 \mu \mathrm{G}$. In any case, we expect fewer than 10 mini-halo candidates detected by LOFAR at $z>0.6$, regardless of the value of the magnetic field.

This argument on the magnetic field is straightforward in the case of a hadronic origin of mini-halos. On the other hand, in reacceleration models the increase of inverse Compton losses with redshift makes reacceleration more difficult. The decline in the expected number of mini-halos in the case of weak fields may therefore be much stronger than predicted in Fig. 5. All these caveats require future theoretical investigations to better exploit future radio surveys.

\section{Discussion and conclusions}

We started to consider the capabilities of future surveys in detecting mini-halos by considering facilities operating at low 

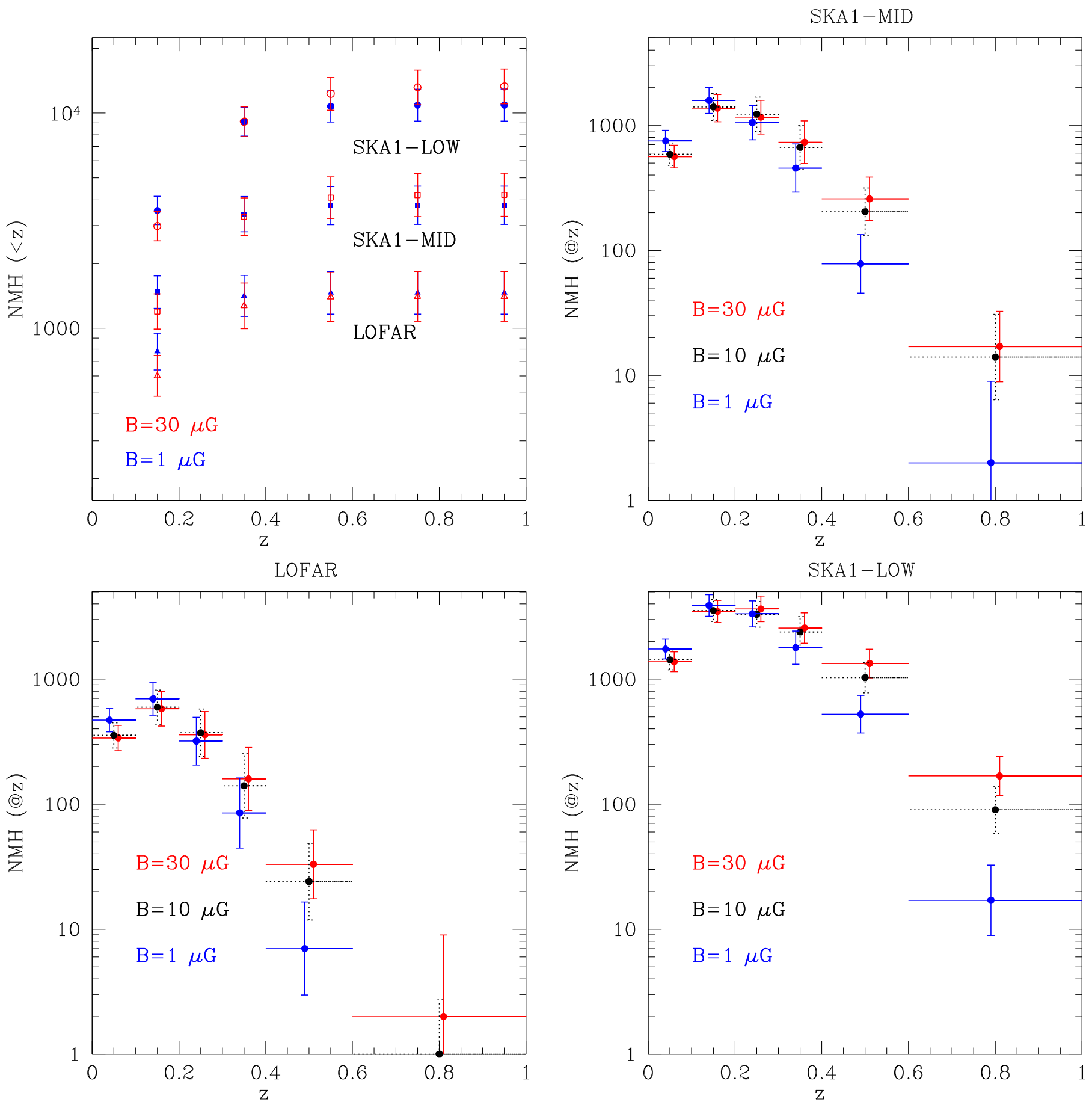

Fig. 5. Top left panel: integral number of radio mini-halo candidates ( $3 \pi$ sr) observable at $140 \mathrm{MHz}$ by LOFAR (triangles) and SKA1-LOW (circles), and at $1.4 \mathrm{GHz}$ by SKA1-MID (squares) as a function of redshift, estimated by assuming two reference values of $B=1 \mu \mathrm{G}$ (blue) and $B=30 \mu \mathrm{G}$ (red). The error bars indicate the $1 \sigma$ uncertainty driven by the $P_{\text {radio }}-L_{\mathrm{X}}$ correlation (Eq. (4)). Top right panel: differential number of radio mini-halo candidates $(3 \pi \mathrm{sr})$ observable at $1.4 \mathrm{GHz}$ by SKA1-MID in different redshift bins, estimated by assuming three reference values of $B=1 \mu \mathrm{G}$ (blue), $B=10 \mu \mathrm{G}$ (black), and $B=30 \mu \mathrm{G}$ (red). The vertical error bars indicate the $1 \sigma$ uncertainty driven by the $P_{\text {radio }}-L_{\mathrm{X}}$ correlation (Eq. (4)), and the horizontal bars indicate the size of the redshift bin. The points corresponding to the three different $B$-field values in the same redshift bin are slightly shifted on the $x$-axis to improve clarity. Bottom left panel: same as top right panel, but showing the results for LOFAR at $140 \mathrm{MHz}$. Bottom right panel: same as top right panel, but showing the results for SKA1-LOW at $140 \mathrm{MHz}$.

(LOFAR, SKA-LOW) and higher (SKA-MID) frequencies. We obtained expectations based on the observed correlation between the radio and X-ray luminosity measured in mini-halo clusters and on its extrapolation toward systems with lower X-ray luminosities and higher redshift. We showed here that future surveys with the SKA and its pathfinder LOFAR have the potential of detecting a large number $\left(\sim 10^{3}-10^{4}\right)$ of radio mini-halo candidates up to redshift $z \sim 1$. This expectation is optimistic because it is based on the assumption that every $\mathrm{CC}$ cluster hosts a minihalo, an assumption that is currently supported only for massive systems at redshift $\leq 0.35$ (Giacintucci et al. 2017). On the other hand, if mini-halos are generated by turbulent reacceleration, 
we might expect a significant drop of their occurrence in less massive CC and at higher redshift. The fraction of nonthermal emission that is channelled into synchrotron radiation declines with redshift in a way that depends on the magnetic field in the mini-halo region. We have shown that this effect generates a decline in luminosity function of mini-halos with redshift and that future surveys with the SKA consequently have the potential of constraining the magnetic field in these systems.

As a caveat for these estimates, we stress that the contamination of point sources, particularly of the central BCG, and the dynamic range of the radio images are important drawbacks for the detection of the diffuse emission of mini-halos at high redshift. However, the SKA is planned to achieve a high dynamic range and to be able to reach angular resolutions (a few arcseconds) that will allow us to accurately subtract the point sources by directly removing their visibilities in the $u v$-plane. Therefore, the residual flux, if present, is assumed to be entirely due to the diffuse emission. The angular size of the mini-halo relative to the resolution of the instrument is therefore not expected to be an issue for the identification of mini-halos.

These caveats are more important at lower frequencies. First, the LOFAR survey LoTSS is being carried out at a nominal resolution of 6 arcsec (Shimwell et al. 2017). This resolution may not be sufficient to accurately subtract discrete sources at higher redshift. Nevertheless, it is also true that higher-resolution images can be generated from the LoTTS data with an appropriate use of the weighting and tapering functions applied to the visibilities during the reduction process, thus controlling the beam shape. Furthermore, the mini-halo candidates selected by the LoTTS could be followed-up at higher resolutions with dedicated, pointed LOFAR observations. Cross-checks with X-ray catalogs will also help to identify AGNs. Second, at low radio frequencies, old bubbles from the central AGNs become more prominent and may provide a significant contamination to the truly diffuse radio emission from the mini-halos. However, these observational issues and the details of the data analysis are beyond the aim of this paper.

We also discussed the synergy of radio surveys with future $\mathrm{X}$-ray missions, showing that a consistent fraction of the clusters selected and characterized by eROSITA will be observable by SKA and LOFAR in various redshift ranges. This will allow us to build large statistical samples of galaxy clusters that can be exploited to study not only the occurrence of radio mini-halos, but also the radio and X-ray properties with an accuracy sufficient to investigate the interplay between nonthermal and thermal cluster components. In particular, according to numerical simulations (ZuHone et al. 2013), turbulence with $\delta v \sim 50-200 \mathrm{~km} \mathrm{~s}^{-1}$ on spatial scales below $\sim 50-100 \mathrm{kpc}$ in the $\mathrm{CC}$ region might be sufficient to reaccelerate seed relativistic electrons producing radio mini-halos. Such turbulence levels are easily detectable with typical exposures of the Athena X-ray Integral Field Unit (X-IFU, Barcons et al. 2017), which is expected to resolve gas velocities of tens of $\mathrm{km} \mathrm{s}^{-1}$ in the cluster cores on 5 arcsec scales (Ettori et al. 2013), corresponding to $\sim 40 \mathrm{kpc}$ at $\mathrm{z} \sim 1$. The synergies of the radio surveys discussed in this work with future Athena X-IFU observations and theoretical studies is essential in establishing the physical nature of radio mini-halos.

Acknowledgements. We would like to thank the anonymous referee for providing constructive comments and raising interesting points that improved the presentation of our work. We acknowledge support from contract ASI-INAF 2017-14-H.0. MG, GB and RC acknowledge support from PRIN-INAF2014.
SE acknowledges the financial contribution from contracts ASI-INAF I/037/12/0, ASI 2015-046-R.0.

\section{References}

Ahnen, M. L., Ansoldi, S., Antonelli, L. A., et al. 2016, A\&A, 589, A33 Akritas, M. G., \& Bershady, M. A. 1996, ApJ, 470, 706

Barcons, X., Barret, D., Decourchelle, A., et al. 2017, Astron. Nachr., 338, 153 Best, P. N., von der Linden, A., Kauffmann, G., Heckman, T. M., \& Kaiser, C. R. 2007, MNRAS, 379, 894

Böhringer, H., Collins, C. A., Guzzo, L., et al. 2002, ApJ, 566, 93

Braun, R., Bourke, T., Green, J. A., Keane, E., \& Wagg, J. 2015, POS(AASKA14) 174

Bravi, L., Gitti, M., \& Brunetti, G. 2016, MNRAS, 455, L41

Brunetti, G., \& Jones, T. W. 2014, Int. J. Mod. Phys. D, 23, 30007

Burns, J. O. 1990, AJ, 99, 14

Cassano, R., Gitti, M., \& Brunetti, G. 2008, A\&A, 486, L31

Cassano, R., Brunetti, G., Norris, R. P., et al. 2012, A\&A, 548, A100

Cassano, R., Ettori, S., Brunetti, G., et al. 2013, ApJ, 777, 141

Cassano, R., Bernardi, G., Brunetti, G., et al. 2015, PoS(AASKA14)073

Cavagnolo, K. W., Donahue, M., Voit, G. M., \& Sun, M. 2009, ApJS, 182, 12

Cavagnolo, K. W., McNamara, B. R., Wise, M. W., et al. 2011, ApJ, 732, 71

Dennis, T. J., \& Chandran, B. D. G. 2005, ApJ, 622, 205

Dunn, R. J. H., \& Fabian, A. C. 2006, MNRAS, 373, 959

Ebeling, H., Edge, A. C., \& Fabian, A. C., et al. 1997, ApJ, 479, L101

Ebeling, H., Edge, A. C., Mantz, A., et al. 2010, MNRAS, 407, 83

Ettori, S., Pratt, G. W., de Plaa, J., et al. 2013, ArXiv e-prints, [arXiv:1306.2322]

Feretti, L., Giovannini, G., Govoni, F., \& Murgia, M. 2012, A\&ARv, 20, 54

Fujita, Y., \& Ohira, Y. 2012, ApJ, 746, 53

Fujita, Y., Matsumoto, T., \& Wada, K. 2004, JKAS, 37, 571

Gaspari, M., Brighenti, F., \& Temi, P. 2012, MNRAS, 424, 190

Gendron-Marsolais, M., Hlavacek-Larrondo, J., van Weeren, R. J., et al. 2017, MNRAS, 469, 3872

Giacintucci, S., Markevitch, M., Brunetti, G., et al. 2014a, ApJ, 795, 73

Giacintucci, S., Markevitch, M., Venturi, T., et al. 2014b, ApJ, 781, 9

Giacintucci, S., Markevitch, M., Cassano, R., et al. 2017, ApJ, 841, 71

Gitti, M., Brunetti, G., \& Setti, G. 2002, A\&A, 386, 456

Gitti, M., Brunetti, G., Feretti, L., \& Setti, G. 2004, A\&A, 417, 1

Gitti, M., Ferrari, C., Domainko, W., et al. 2007, A\&A, 470, L25

Gitti, M., Brighenti, F., \& McNamara, B. R. 2012, Adv. Astron., 2012

Gitti, M., Tozzi, P., Brunetti, G., et al. 2015, PoS(AASKA14)076

Govoni, F., Murgia, M., Markevitch, M., et al. 2009, A\&A, 499, 371

Heinz, S., Brüggen, M., Young, A., \& Levesque, E. 2006, MNRAS, 373, L65

Hitomi Collaboration, (Aharonian, F., et al.) 2016, Nature, 535, 117

Hitomi Collaboration, (Aharonian, F., et al.) 2018, PASJ, 70, 9

Hudson, D. S., Mittal, R., Reiprich, T. H., et al. 2010, A\&A, 513, A37

Ignesti, A., Gitti, M., Brunetti, G., Feretti, L., \& Giovannini, G. 2017, A\&A, 604, A21

Jacob, S., \& Pfrommer, C. 2017, MNRAS, 467, 1478

Mazzotta, P., \& Giacintucci, S. 2008, ApJ, 675, L9

McDonald, M., Bayliss, M., Benson, B. A., et al. 2012, Nature, 488, 349

Mittal, R., Hudson, D. S., Reiprich, T. H., \& Clarke, T. 2009, A\&A, 501, 835

Mullis, C. R., Vikhlinin, A., Henry, J. P., et al. 2004, ApJ, 607, 175

Murgia, M., Govoni, F., Markevitch, M., et al. 2009, A\&A, 499, 679

Pfrommer, C., \& Enßlin, T. A. 2004, A\&A, 413, 17

Piffaretti, R., Arnaud, M., Pratt, G. W., Pointecouteau, E., \& Melin, J.-B. 2011, A\&A, 534, A109

Pillepich, A., Porciani, C., \& Reiprich, T. H. 2012, MNRAS, 422, 44

Prandoni, I., \& Seymour, N. 2015, PoS(AASKA14)067

Reiprich, T. H., \& Böhringer, H. 2002, ApJ, 567, 716

Samuele, R., McNamara, B. R., Vikhlinin, A., \& Mullis, C. R. 2011, ApJ, 731,31

Santos, J. S., Tozzi, P., Rosati, P., \& Böhringer, H. 2010, A\&A, 521, A64

Shimwell, T. W., Röttgering, H. J. A., Best, P. N., et al. 2017, A\&A, 598, A104

Sommer, M. W., Basu, K., Intema, H., et al. 2017, MNRAS, 466, 996

van Haarlem, M. P., Wise, M. W., Gunst, A. W., et al. 2013, A\&A, 556, A2

van Weeren, R. J., Intema, H. T., Lal, D. V., et al. 2014, ApJ, 786, L17

Zandanel, F., Pfrommer, C., \& Prada, F. 2014, MNRAS, 438, 124

Zhuravleva, I., Churazov, E., Schekochihin, A. A., et al. 2014, Nature, 515,85

Zhuravleva, I., Churazov, E., Arévalo, P., et al. 2016, MNRAS, 458, 2902

ZuHone, J. A., Markevitch, M., Brunetti, G., \& Giacintucci, S. 2013, ApJ, 762,78 